\title{
ARTICLE OPEN Tunable quantum critical point and detached superconductivity in Al-doped CrAs
}

\author{
Sungmin Park ${ }^{1}$, Soohyeon Shin ${ }^{1}$, Sung-II Kim ${ }^{1}$, Suyoung Kim ${ }^{1}$, Chan-Koo Park ${ }^{1}$, Joe D. Thompson ${ }^{2}$ and Tuson Park ${ }^{1}$
}

The origin of unconventional superconductivity and its relationship to a $T=0 \mathrm{~K}$ quantum critical point (QCP), which is hidden inside the dome of a superconducting state, have long been an outstanding puzzle in strongly correlated superconductors. The observation and tuning of the hidden $\mathrm{QCP}$, which is key to resolving the mystery, however, has been rarely reported. Here we report the controlling of a hidden QCP in the helical antiferromagnet CrAs and separation of the tuned QCP from the pressureinduced superconducting phase. The Al doping in $\mathrm{CrAs}$ increases the antiferromagnetic ordering temperature $T_{\mathrm{N}}$ from 265 to $275 \mathrm{~K}$, while it suppresses the QCP from 8 to $4.5 \mathrm{kbar}$. Pressure-induced superconductivity in the high-pressure regime is almost independent of Al doping, but superconductivity below $6 \mathrm{kbar}$ is suppressed, revealing the clear separation between the tuned antiferromagnetic QCP and $T_{\mathrm{c}}$ maximum. These discoveries illustrate subtleties in the interplay between superconductivity and quantum criticality and warrant a deeper insight in understanding of unconventional superconductivity.

npj Quantum Materials (2019)4:49; https://doi.org/10.1038/s41535-019-0188-6

\section{INTRODUCTION}

Unconventional superconductivity commonly emerges in proximity to a magnetically ordered phase, raising the possibility that critical spin fluctuations may mediate the formation of superconducting (SC) Cooper pairs. ${ }^{1-10}$ In the vast majority of these cases, as sketched in Fig. 1a, the zero-temperature limit of a continuous magnetic phase boundary (a quantum critical point $(\mathrm{QCP})$ ) is veiled by a dome of superconductivity, making it difficult to prove the interplay between unconventional superconductivity and fluctuations arising from the presumed QCP. Nevertheless, unusual normal state properties of these materials above their dome of superconductivity imply that a magnetic QCP may remain a viable concept even below $T_{c}$ in various classes of unconventional superconductors, such as those based on Fe and $\mathrm{Cu}$ as well as heavy fermion compounds, in which there is an intricate interplay among intertwined order parameters. ${ }^{11-14}$ More definitive evidence for the connection between quantum criticality and unconventional superconductivity may come from experiments showing that an SC phase is pinned to or detached from tunable QCPs. ${ }^{15}$ When a QCP is moved, as sketched in Fig. $1 \mathrm{~b}$, the SC phase will be pinned to the tuned QCP if Cooper pairing is produced by the critical quantum fluctuations, while it can detach from the QCP if the two phenomena are independent of each other, therefore providing a stringent test to resolve the potential relationship between QCP and unconventional SC state in strongly correlated systems.

$\mathrm{CrAs}$, with $\mathrm{MnP}$ orthorhombic structure, orders in a non-collinear helimagnetic structure below $T_{\mathrm{N}}=260 \mathrm{~K}$, which is accompanied by a discontinuous lattice expansion along the crystalline $b$-axis and contraction along $a$ - and $c$-axes. ${ }^{16-18}$ The crystal structure remains unchanged through the transition but its cell volume, dominated by expansion along $b$, is larger below $260 \mathrm{~K}$. With initial applied pressure, the coupled magnetic and structural transitions move to lower temperatures. Though weak diamagnetism and zero resistance appear already at pressures near $3 \mathrm{kbar}$, the highest $T_{c}$ occurs near the critical pressure of $8 \mathrm{kbar}$ where the coupled magnetic/structural transition is projected to zero Kelvin and electrical resistivity deviates from the Landau-Fermi liquid $T^{2}$ dependence, indicating a helical antiferromagnetic (AFM) QCP hidden below the dome of pressure-induced superconductivity in CrAs. ${ }^{19-22}$ Recent neutron scattering suggests that the non-Fermi liquid behavior arises from a nearly second-order helical magnetic phase transition that is accompanied by a first-order isostructural transition. ${ }^{22}$ Even though these experiments provide circumstantial evidence for a close relationship between quantum criticality and superconductivity, the complexity of simultaneous magnetic and isostructural transitions and the possibility of electronic phase separation in polycrystalline $\mathrm{CrAs}^{23}$ cloud a straightforward connection between criticality and superconductivity.

Here we show that a projected critical point in CrAs is successfully shifted by Al chemical substitution and the pressure-induced SC phase is detached from the tuned magnetic critical point. Slight Aldoping increases $T_{\mathrm{N}}$ from $265 \mathrm{~K}$ to $275 \mathrm{~K}$ but pressure rapidly suppresses $T_{\mathrm{N}}$ of $\mathrm{Al}-\mathrm{CrAs}$ to zero Kelvin near $4.5 \mathrm{kbar}\left(=P_{\mathrm{C}}\right.$ ), giving a suppression rate that is nearly two times faster than that of pure CrAs. The residual resistivity as well as the temperature coefficient of resistivity peak near $P_{C}$, indicating that the projected critical point is shifted from $8 \mathrm{kbar}$ for pure $\mathrm{CrAs}$ to $4.5 \mathrm{kbar}$ by Al doping. Contrary to the tunable critical point, the maximum $T_{c}$ of pressure-induced SC remains near $8 \mathrm{kbar}$, showing that the SC dome is detached from the shifted QCP. These discoveries evidence that superconductivity in CrAs is produced in spite of the QCP, not because of it. The unambiguous demonstration of detached superconductivity from the QCP in Al-CrAs illustrates that tuning via non-thermal control

\footnotetext{
${ }^{1}$ Center for Quantum Materials and Superconductivity (CQMS) \& Department of Physics, Sungkyunkwan University, Suwon 16419 , South Korea and ${ }^{2}$ Los Alamos National Laboratory, Los Alamos, NM 87545, USA

Correspondence: Tuson Park (tp8701@skku.edu)

These authors contributed equally: Sungmin Park, Soohyeon Shin
}

Received: 30 March 2019 Accepted: 15 August 2019

Published online: 05 September 2019 

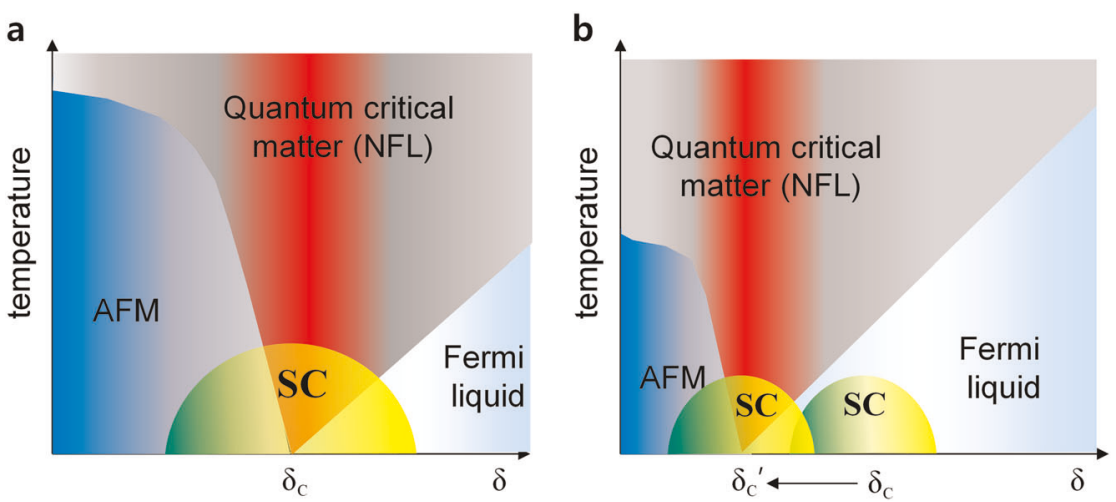

Fig. 1 Schematic temperature-control parameter $(T-\delta)$ phase diagram of superconductivity and quantum critical matter. a Unconventional superconductivity is often observed in proximity to a projected quantum critical point $\left(\delta_{c}\right)$, where non-Fermi liquid behavior is observed in the normal state above the SC phase. b When $\delta_{c}$ is tuned to a new point $\delta_{c}{ }^{\prime}$, the SC phase can be either moved to the new $\delta_{c}{ }^{\prime}$ or remain at the old $\delta_{c}$ - see the main text for discussion
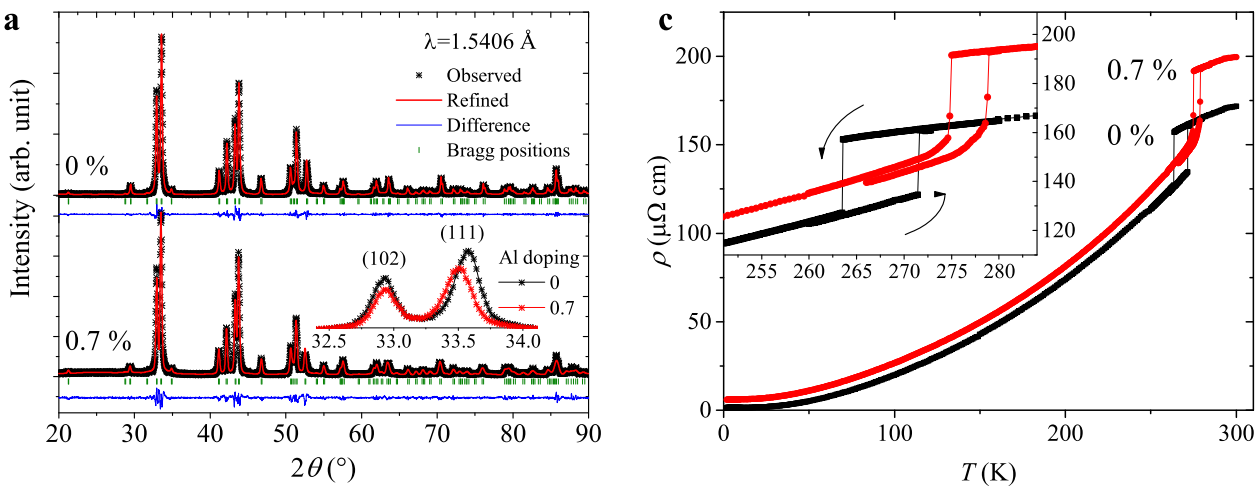

b
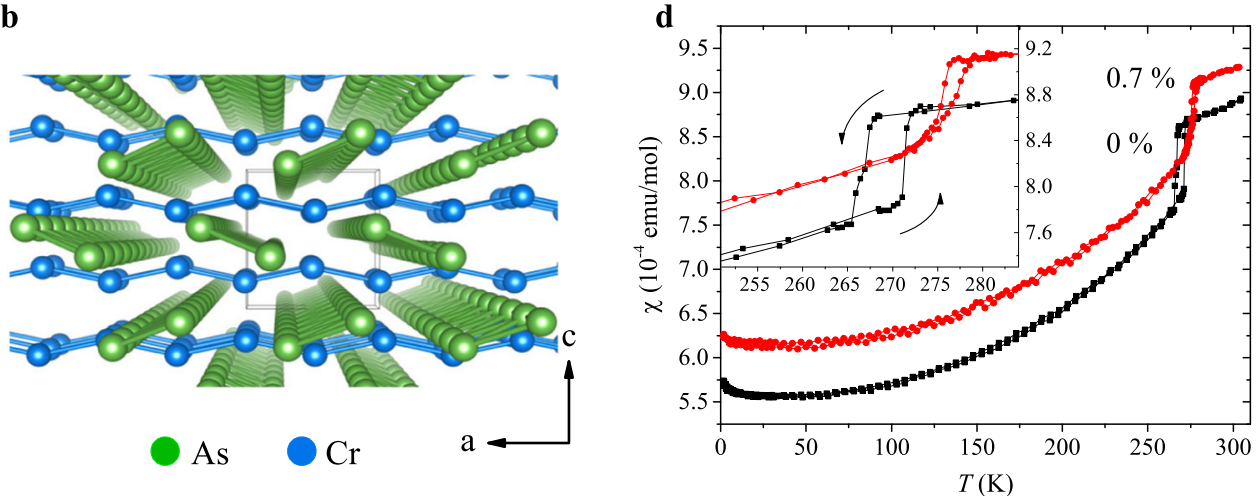

Fig. 2 Powder X-ray diffraction and physical properties at ambient pressure. a Powder X-ray diffraction patterns of pure and $0.7 \pm 0.24 \%$ Aldoped CrAs in upper and lower panels, respectively. Inset to lower panel: magnified view near (102) and (111) peaks for both pure and Aldoped CrAs. b Schematic crystal structure of $\mathrm{CrAs}$, where As and $\mathrm{Cr}$ atoms are depicted as green and blue spheres. $\mathbf{c}$ Electrical resistivity $\rho$ of pure (black symbols) and Al-doped CrAs (red symbols) for electrical current applied along the crystalline $a$-axis. The inset is an expanded view of $\rho$ near the transition temperature. $\mathbf{d}$ Magnetic susceptibility $(X)$ of pure (black symbols) and Al-doped CrAs (red symbols). The inset plots $X(T)$ near the AFM transition

parameters can provide an alternative route to probe the intricate relationship between a hidden critical point and surrounding superconductivity.

\section{RESULTS AND DISCUSSION}

Figure 2a shows the powder X-ray diffraction (PXRD) patterns of pure and Al-doped CrAs crystals. Rietveld refinement of the pattern matches the peak positions and intensities of the orthorhombic MnP-type crystal structure (space group Pnma, 62 ), indicating that the single crystals are in a single-phase without any detectable impurity phases. The lattice constants change from $a=5.6510, b=3.4688$ and $c=6.2067 \AA$ for pure $\mathrm{CrAs}$ to 5.6499 , 3.4831 and $6.2049 \AA$ for Al-doped CrAs, showing that the $b$-axis lattice constant is elongated and cell volume increased by about $0.4 \%$ with Al doping. Energy-dispersive X-ray spectroscopy (EDS) shows that the $\mathrm{Al}$ concentration in $\mathrm{Al}-\mathrm{CrAs}$ is $0.7 \pm 0.24 \%$, where the error is the standard deviation estimated by taking into account instrumental resolution of EDS and statistical variation in EDS spectra over various spots on the crystal.

The $a$-axis electrical resistivity $\rho$ of Al-doped $\mathrm{CrAs}$ is compared to that of pure CrAs in Fig. 2c. With decreasing temperature, there 
is a sharp drop with hysteresis at 265 and $275 \mathrm{~K}\left(=T_{\mathrm{N}}\right)$ for pure and Al-doped CrAs, respectively, which arises from the coincidence of AFM and isostructural volume expansion transitions. ${ }^{19,20}$ Aluminum substitution not only increases $T_{\mathrm{N}}$ by $10 \mathrm{~K}$, but also increases $\rho$ at $290 \mathrm{~K}$ from 169 to $197 \mu \Omega \mathrm{cm}$, due in part to the increase in disorder and higher transition temperature. Likewise, the residual resistivity $\rho_{0}$, estimated by extrapolating $\rho(T)$ from base temperature to $0 \mathrm{~K}$, increases from 1.4 to $6.0 \mu \Omega \mathrm{cm}$ and the residual resistivity ratio (RRR) decreases from 120 to 33, again signifying that disorder from $\mathrm{Al}$ substitution contributes to electron scattering. Concomitant with the resistivity results, as shown in Fig. 2d, magnetic susceptibility measurements find that $T_{\mathrm{N}}$ increases from $265 \mathrm{~K}$ for pure $\mathrm{CrAs}$ to $275 \mathrm{~K}$ for $\mathrm{Al}-\mathrm{CrAs}$, demonstrating that $\mathrm{Al}$ substitution is of bulk nature.

Though the larger cell volume of Al-CrAs is consistent with its higher magnetic/structural transition temperature, Al- doping is not a simple negative chemical pressure effect. Figure $3 a, b$ comparatively shows the pressure dependence of the magnetic and SC phase transition temperatures of Al-doped and pure $\mathrm{CrAs}$, respectively. $T_{\mathrm{N}}$ of Al-CrAs decreases gradually with initial pressure but drops rapidly for pressures higher than 4 kbar, similar to what happens in pure CrAs near $7 \mathrm{kbar}$. If $\mathrm{Al}$ were acting solely as a negative chemical pressure, the crossover to a steep decrease in $T_{\mathrm{N}}$ should occur at a higher pressure in $\mathrm{Al}-\mathrm{CrAs}$. Nevertheless, a smooth extrapolation of $T_{\mathrm{N}}(P)$ indicates that the magnetic transition reaches $T=0$ near $4.5 \mathrm{kbar}\left(=P_{\mathrm{C}}\right)$, which is nearly half the critical pressure of $8 \mathrm{kbar}$ for $\mathrm{CrAs}$, even though its $T_{\mathrm{N}}$ is $10 \mathrm{~K}$ lower.

The increase in $T_{\mathrm{N}}$ from 265 to $275 \mathrm{~K}$ with Al-doping might reasonably be expected from a negative pressure effect because of the larger cell volume and particularly expanded $b$-axis. Since the $\mathrm{Cr}-3 \mathrm{~d}$ states are more localized due to reduction in $\mathrm{p}-\mathrm{d}$ mixing between $\mathrm{Cr} 3 \mathrm{~d}$ and the anion $\mathrm{p}$ states, the already sizeable ordered moment $\left(1.73 \mu_{\mathrm{B}}\right)$ in $\mathrm{CrAs}$ should increase with Al doping as should $T_{\mathrm{N}}{ }^{24}$ The substantially lower $P_{\mathrm{C}}$, however, indicates that the effect of Al-doping cannot be explained simply by a reduction in $\mathrm{p}-\mathrm{d}$ mixing. As with itinerant antiferromagnetism in $\mathrm{V}$-doped $\mathrm{Cr}$, pressure and chemical doping play very different roles due both to impurity scattering and to changes in the electronic structure. $^{25,26}$ This is likely as well to be the case in Al-CrAs, where $\mathrm{Al}$ introduces both disorder and additional carriers as $\mathrm{V}$ does in $\mathrm{Cr}$. Interestingly, a few atomic percent $\mathrm{V}$ in $\mathrm{Cr}$ also substantially decreases the critical pressure of AFM order and induces a very rapid drop in $T_{\mathrm{N}}(P)$ as the critical pressure is approached. Clearly, experimental and theoretical studies of the pressure-dependent electronic structure will be important to understand the microscopic role of Al-doping in $\mathrm{CrAs}$.

Color contour plots of the temperature and pressure variation in $\rho$ of Al-doped and pure $\mathrm{CrAs}$ are, respectively, depicted in Fig. 3a, $\mathrm{b}$ on a semi-logarithmic scale, where the raw data are plotted in Fig. S1 in the Supplementary Information (SI). Magnetic/structural transition temperatures overlaid in the contour map are determined from a peak in $\mathrm{d} \rho / \mathrm{d} T$ (see Fig. $\mathrm{S} 2$ in the $\mathrm{SI}$ ). The low- $T$ resistivity contour of $\mathrm{CrAs}$, illustrated in Fig. 3b, does not show an anomalous behavior except for a slight change in the slope of the resistivity contour near $8 \mathrm{kbar}$. In contrast, the large scattering region marked by red in $\mathrm{Al}$-doped $\mathrm{CrAs}$, as shown in Fig. 3a, forms a narrow funnel that emerges from the projected $T=0 \mathrm{~K}$ critical point at $4.5 \mathrm{kbar}\left(=P_{\mathrm{C}}\right)$. When the system is away from the critical pressure, the electron scattering rate at low temperatures becomes smaller, indicating that the anomalously large scattering rate at $P_{C}$ arises from the critical magnetic fluctuations associated with the quantum phase transition.

Figure $4 a$ gives the pressure evolution of the low-temperature resistivity of Al-CrAs, where $\rho$ is plotted as a function of $T$ and shifted rigidly with an offset at each pressure for clarity. The dashed red lines are least-squares fits to a power-law behavior, i.e., $\rho=\rho_{0}+A T^{n}$, where the best results of residual resistivity $\rho_{0}$ and
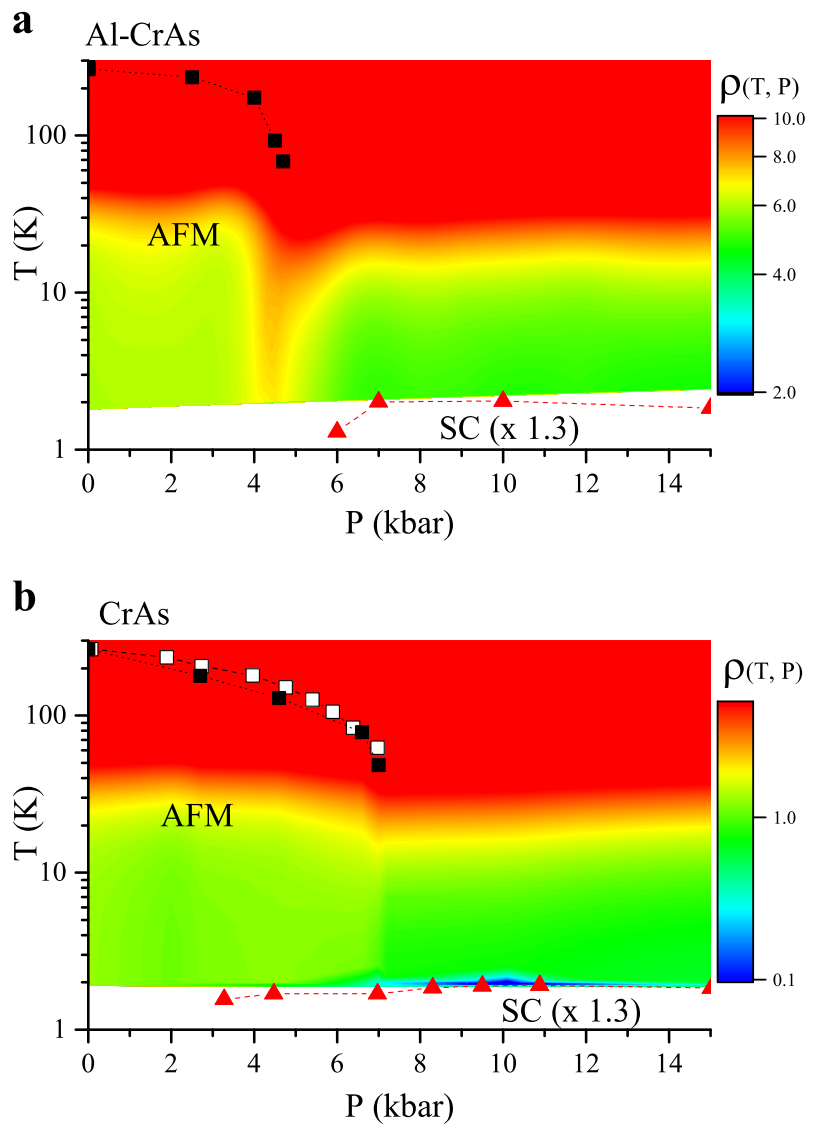

Fig. 3 Three-dimensional contour map of electrical resistivity of Aldoped $\mathrm{CrAs}$ in $\mathbf{a}$ and pure $\mathrm{CrAs}$ in $\mathbf{b}$. The magnitude of the electrical resistivity is described by false colors in the temperature-pressure plane. a Anomalously enhanced electron scattering (red color) forms a funnel shape near the projected critical pressure of $4.5 \mathrm{kbar}$ for Al-CrAs. Sold squares represent $T_{N}$, while solid triangles describe the SC transition temperature $T_{\mathrm{c}}$. $\mathbf{b} T_{\mathrm{N}}$ of CrAs is described by solid and open squares that are data taken from this work and ref. ${ }^{19}$ respectively. Solid triangles represent $T_{c}$ of $\mathrm{CrAs}$ that are taken from ref. ${ }^{19} ; T_{c}$ of $\mathrm{CrAs}$ and $\mathrm{Al}-\mathrm{CrAs}$ are multiplied by 1.3 for comparison. Dotted lines connected with AFM transitions are guides to the eyes. AFM and SC stands for antiferromagnetic and superconducting phase, respectively

coefficient $A$ are plotted on the left and right ordinates of Fig. $4 \mathrm{~b}$, respectively. At lower pressures $\left(P<P_{\mathrm{C}}\right), n$ is close to 2 , as expected for Landau-Fermi liquid behavior. With increasing pressure, the exponent $n$ of $\mathrm{Al}-\mathrm{CrAs}$ sharply drops close to 1.5 at $4.5 \mathrm{kbar}$ and gradually increases to 1.83 at $24.1 \mathrm{kbar}$. The non-Fermi liquid behavior can be ascribed to scattering by critical fluctuations associated with the AFM QCP at $P_{\mathrm{C}}$. Underpinning the presence of the QCP, $\rho_{0}$ shows a sharp peak and $A$ abruptly increases by a factor of 30 at the critical pressure $P_{C}(=4.5 \mathrm{kbar})$, as shown in Fig. $4 b$, c. In pure CrAs, analysis of the low- $T$ resistivity by powerlaw fits also shows that the non-Fermi liquid behavior appears near $8 \mathrm{kbar}$, the pressure across which there occurs a change in the slope of resistivity, and the critical region extends over a wide pressure range $(P>8 \mathrm{kbar})$-see Fig. S3 in the SI. The fact that non-Fermi liquid behavior is observed near the tuned QCP of AlCrAs underscores that the strange metallic behavior near the optimal pressure $\left(P_{c}\right)$ in $\mathrm{CrAs}$ originates from critical magnetic fluctuations associated with the AFM QCP veiled by the dome of SC phase.

The SC transition temperature $T_{c}$, defined by the $90 \%$ resistance drop from that at $T_{c}$ onset in Al-doped CrAs (see Fig. S4 and evidence for bulk superconductivity in Fig. $\mathrm{S} 5$ of the $\mathrm{SI}$ ), starts to 

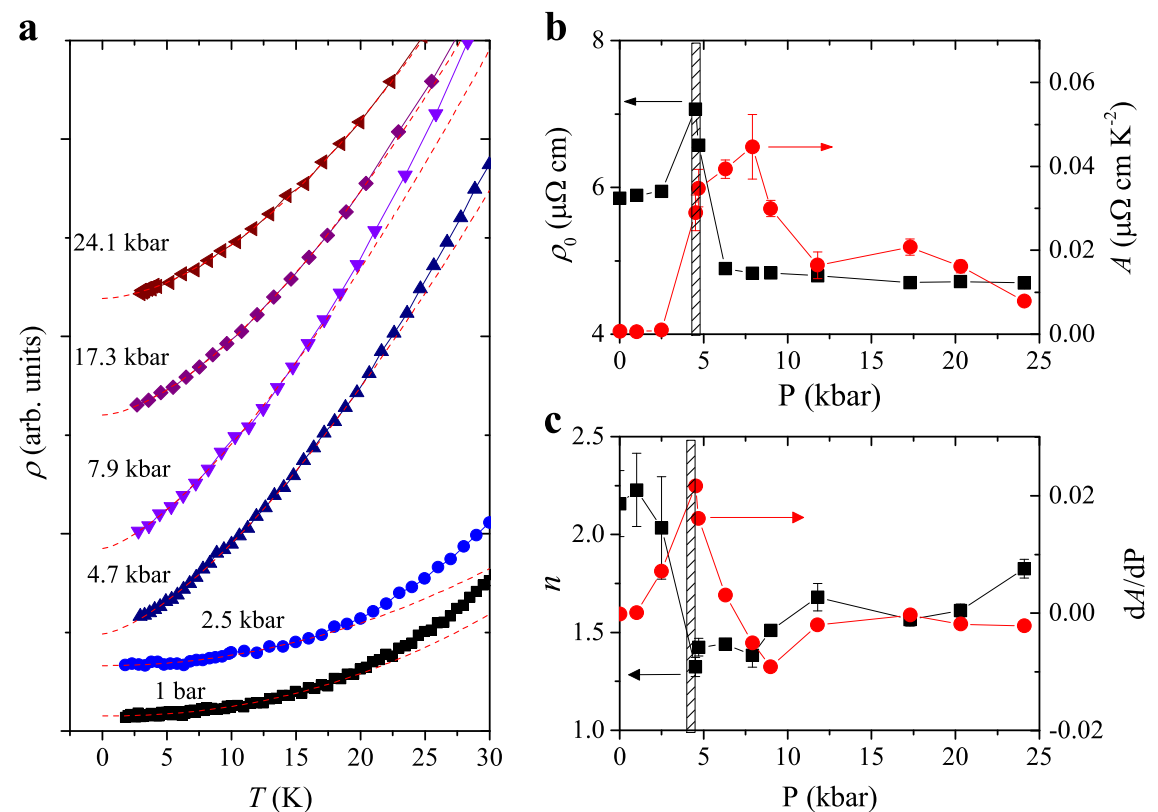

Fig. 4 Low-temperature electrical resistivity of Al-doped CrAs under pressure. a Electrical resistivity ( $\rho$ ) of Al-doped CrAs is plotted against $T$ for several pressures. For clarity, $\rho$ at each pressure is shifted rigidly by an offset. Solid lines are least-squares fits to a power-law form, i.e. $\rho=$ $\rho_{0}+A T^{n}$, where the fitted values for $\rho_{0}$ (squares) and $A$ (circles) are plotted as a function of pressure on the left and right ordinates in $\mathbf{b}$, respectively. c The exponent $n$ (solid squares) is plotted on the left ordinate and the first derivative of the coefficient $A$ with respect to pressure $(P), \mathrm{d} A / \mathrm{d} P$ (solid circles), is shown on the right ordinate, where it is peaked near 4.5 kbar. Error bars describe the standard deviation from the least-squares fitting

appear for pressures above the critical pressure $P_{\mathrm{C}}$ (=4.5 kbar), as marked by the triangles in Fig. 3a. With further increasing pressure, $T_{c}$ reaches a maximum near $10 \mathrm{kbar}$, and gradually decreases to $1.27 \mathrm{~K}$ at $24.1 \mathrm{kbar}$. When compared with pure CrAs (see Fig. $3 \mathrm{~b}$ ), where $T_{\mathrm{c}}$ starts to appear at $3 \mathrm{kbar}$, the pressure required to induce an initial zero-resistance state is shifted to a higher pressure in Al-CrAs. However, the pressure-dependent dome of $T_{c}(P)$ is similar to that of pure CrAs in that a broad maximum appears near $10 \mathrm{kbar}$ for both compounds. We note that the zero-resistance SC state starts to appear deep in the AFM state for pure CrAs, while it exists only after the AFM phase is completely suppressed for Al-CrAs.

The superconductivity detached from a QCP in Al-CrAs is in stark contrast with those unconventional superconductors where non-Fermi liquid behaviors appear above the optimal doping or pressure at which the highest $T_{\mathrm{c}}$ appears. Superconductivity in pure CrAs has been proposed to be mediated by magnetic fluctuations because of its proximity to a possible AFM QCP. ${ }^{19-21}$ The absence of a coherence peak in the spin-lattice relaxation rate $T_{1}$ and a $T^{3}$ dependence of $1 / T_{1}$ below $T_{c}$ are consistent with unconventional superconductivity in CrAs. ${ }^{21}$ The observations of both six-fold and two-fold symmetric components in the fieldangle dependent upper critical field of CrAs support this conclusion and further argue for odd-parity spin-triplet pairing. ${ }^{27}$ When $\mathrm{CrAs}$ is doped with $\mathrm{Al}$, however, the maximum $T_{\mathrm{c}}$ at the optimal pressure near $10 \mathrm{kbar}$ is independent of the disorder even though the residual resistivity increases from 1.4 to $6.0 \mu \Omega \mathrm{cm}$ with Al doping. This robustness of the superconductivity against introduction of non-magnetic impurities seems at odds with simple triplet superconductivity that is easily destroyed by any type of impurities. ${ }^{28}$ Further, muon-spin rotation measurements of CrAs under pressure find scaling of the superfluid density, $n_{\mathrm{s}} \propto T_{\mathrm{c}}^{3.2}$, which is consistent with conventional phonon pairing. ${ }^{23}$ The peculiar band structure of CrAs, where its possible non-trivial band crossing is protected by the non-symmorphic crystal structure, may be important in unraveling the mechanism and nature of superconductivity in this fascinating material. ${ }^{23,29}$ Additional study that can give direct information on the SC gap, such as point contact spectroscopy and field-directional specific heat measurements under pressure, will be important to resolve these contradicting results.

Figure $5 \mathrm{a}$ and $\mathrm{b}$ sequentially describes a color contour map of normalized isothermal resistivity $(\mathrm{n} \rho)$ in the $T-P$ plane for pure and Al-doped $\mathrm{CrAs}$, where electrical resistivity $\rho(P)$ was divided by $\rho$ at the highest measuring pressure for comparison, e.g., $\mathrm{n} \rho=\rho(P) / \rho$ ( $24.1 \mathrm{kbar})$ for Al-CrAs. The representative raw data that Fig. 5 is based on are plotted in Fig. S6 in the SI. In the high- $T$ regime, strong electron scattering (red) is observed mainly outside the magnetic phase boundary due to thermally induced critical fluctuations for both compounds. In the low-T regime of $\mathrm{CrAs}$, as shown in Fig. 5b, the area of enhanced scattering extends over the high-pressure regime $\left(P>P_{c}\right)$, which is consistent with recent neutron scattering and NQR results that showed abundant magnetic fluctuations in the normal state near the $T_{\mathrm{c}}$ maximum pressure. $^{21,22}$ There occurs a rapid change in the resistivity across the critical pressure of $8 \mathrm{kbar}$. The suppression of $T_{\mathrm{c}}$ at this critical pressure is indicative of a competition between SC and the double helical magnetic phase. On the other hand, the low- $T$ isothermal resistivity of $\mathrm{Al}-\mathrm{CrAs}$ in Fig. 5a shows a disparate behavior, forming a funnel of strongly enhanced electron scattering in the vicinity of the critical pressure of $4.5 \mathrm{kbar}$. The enhanced scattering decreases gradually with increasing the distance below and above $P_{\mathrm{C}}$ (see Fig. S6a in the SI). The fact that the transition width becomes broader at lower temperatures is in contrast to a classical phase transition where the phase transition becomes sharper with decreasing temperature due to reduction in thermal fluctuations. The lambda-like enhanced resistivity across the critical pressure indicates that nature of the magnetic transition is weakly first order or second order at lower temperatures.

The pressure evolution of the double helical magnetic phase of $\mathrm{CrAs}$, which is important to shed light on the nature of superconductivity, has yet to be elucidated. ${ }^{16,17,21,22}$ Shen et al. performed neutron diffraction on a polycrystalline sample and reported a spin reorientation transition from the $a b$ plane to the 
a
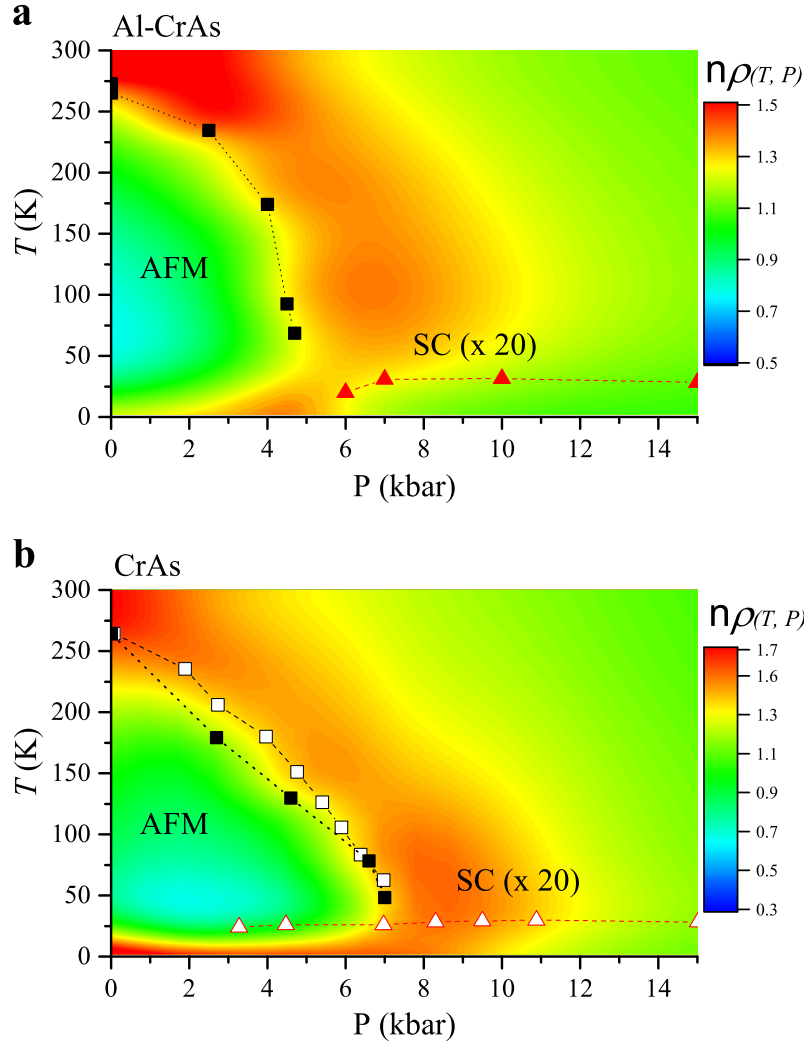

Fig. 5 Three-dimensional contour map of normalized isothermal electrical resistivity $(n \rho)$ as a function of pressure for Al-CrAs and $\mathrm{CrAs}$ in $\mathbf{a}$ and $\mathbf{b}$, respectively. For comparison, isothermal electrical resistivity of $\mathrm{Al}-\mathrm{CrAs}$ is divided by $\rho$ at $24.1 \mathrm{kbar}$, while that of $\mathrm{CrAs}$ is divided by $\rho$ at $22.5 \mathrm{kbar}$, the highest measuring pressures for both compounds. Raw data on which the contour plot is based are plotted in Fig. S6 in the SI. Squares and triangles represent $T_{\mathrm{N}}$ and $T_{\mathrm{C}}$ respectively, where $T_{\mathrm{c}}$ is multiplied by 20 for comparison. AFM and SC stands for antiferromagnetic and superconducting phase, respectively

ac plane at $6 \mathrm{kbar}$, whereas the pressure-induced magnetic phase disappears near 9.4 kbar. $^{17}$ In contrast, Matsuda et al. studied neutron diffraction on a single crystal $\mathrm{CrAs}$ under hydrostatic pressure and reported that the helical magnetic order completely disappears at 6.9 kbar. $^{22}$ This discrepancy on the spin reorientation transition was then ascribed to the usage of single crystals in their study. As shown in Fig. 5b, enhanced resistivity extends across the critical pressure of $8 \mathrm{kbar}$, but it is difficult to find any clear signature that may indicate the presence of an additional phase transition near $9.4 \mathrm{kbar}$ - see also Fig. S6b in the Sl. Unlike pure CrAs, the enhanced isothermal resistivity in Al-CrAs is not extended above the critical pressure of $4.7 \mathrm{kbar}$, instead it is mostly confined near $P_{c}$ in Fig. 5a. Absence of any clear signature at higher pressure indicates that it is unlikely to have an additional phase transition in Al-CrAs. In order to make a definitive statement on the spin reorientation transition in $\mathrm{Al}-\mathrm{CrAs}$, however, further work such as neutron scattering or NQR experiments under pressure is necessary.

Observation of a QCP veiled by a dome of SC phase has been key in interpreting the origin of non-Fermi liquid behaviors in the normal state, coexistence of competing phases, and unconventional superconductivity for various classes of correlated superconductors. By synthesizing $0.7 \pm 0.24 \%$ Al-doped CrAs single crystals whose AFM phase transition and isostructural volume expansion occur at $275 \mathrm{~K}$, a $10 \mathrm{~K}$ increase from $265 \mathrm{~K}$ in pure $\mathrm{CrAs}$, we successfully moved a critical point from 8 to $4.5 \mathrm{kbar}$. Observation of a funnel of enhanced electron scattering and the
non-Fermi liquid behavior at the critical pressure is consistent with a magnetic QCP at that pressure for Al-doped CrAs. The SC phase, in contrast, forms a dome as a function of pressure with a maximum $T_{\mathrm{c}}$ near $10 \mathrm{kbar}$, very similar to the case of pure CrAs. The superconductivity detached from the QCP in Al-doped CrAs is different from pure CrAs and suggests that Cooper pair formation is not mediated solely, or if at all, by critical magnetic fluctuations. These discoveries not only point to new directions and needs for future theory and experiment work on $\mathrm{CrAs}$ but also indicate more broadly the power of using multiple non-thermal tuning parameters simultaneously to reveal the relationship between superconductivity and a hidden QCP in classes of unconventional superconductors.

\section{METHODS}

Single crystals of pure $\mathrm{CrAs}$ and $\mathrm{CrAs}$ doped with $\mathrm{Al}$ were grown out of a Sn-flux, as described elsewhere. ${ }^{30}$ PXRD was measured with a Rigaku miniflex-600 (Cu K-a source, $\lambda \sim 1.5406 \AA$ ) and the data were refined in the Fullprof program to determine the lattice constants at room temperature. A conventional four-probe technique was applied to measure electrical resistivity of needle-shaped $\mathrm{CrAs}$ with current flow in the needle along its elongated crystalline $a$-axis. At ambient pressure, the RRR of Al-CrAs is $\sim 33$, which is lower than that of pure $\mathrm{CrAs}$. Pressure measurements to $24.1 \mathrm{kbar}$ were performed in a hybrid Be-Cu/NiCrAl clamp-type pressure cell with silicone oil as the pressure medium. The pressure-dependent SC transition temperature of a $\mathrm{Pb}$ manometer was used to determine the pressure. ${ }^{31}$ Resistivity under pressure was measured in closed cycle refrigerator (CCR) and ${ }^{3} \mathrm{He}$ refrigerators for relatively high- $(2.8-305 \mathrm{~K})$ and low-temperature ranges $(0.25-4 \mathrm{~K})$, respectively. Magnetic susceptibility measurements were carried out in a Magnetic Property Measurement System (MPMS, Quantum Design, Inc.) in an applied field of $5 \mathrm{kOe}$

\section{DATA AVAILABILITY}

The data sets generated and/or analyzed in this study are available from the corresponding author upon request.

\section{ACKNOWLEDGEMENTS}

This work was supported by a National Research Foundation (NRF) of Korea grant funded by the Korean Ministry of Science, ICT, and Planning (No. 2012R1A3A2048816). Work at Los Alamos National Laboratory was performed under the auspices of the U.S. Department of Energy, Office of Basic Energy Sciences, Division of Materials Sciences and Engineering.

\section{AUTHOR CONTRIBUTIONS}

T.P. proposed and supervised the study. S.S. and C.P. provided the samples, and S.P., S.S., S.Y.K. and S.K. performed the measurements. S.P., S.S. and T.P. analysed the data, and S.P., S.S., J.D.T. and T.P. wrote the manuscript with input from all authors.

\section{ADDITIONAL INFORMATION}

Supplementary information accompanies the paper on the npj Quantum Materials website (https://doi.org/10.1038/s41535-019-0188-6).

Competing interests: The authors declare no competing interests.

Publisher's note: Springer Nature remains neutral with regard to jurisdictional claims in published maps and institutional affiliations.

\section{REFERENCES}

1. Mathur, N. D. et al. Magnetically mediated superconductivity in heavy fermion compounds. Nature 394, 39-43 (1998).

2. Stewart, G. R. Non-Fermi-liquid behavior in d-and f-electron metals. Rev. Mod. Phys. 73, 797-855 (2001).

3. Yuan, H. Q. et al. Superconducting phases in $\mathrm{CeCu}_{2} \mathrm{Si}_{2}$. Science 302, 2104-2107 (2003).

4. Park, T. et al. Hidden magnetism and quantum criticality in the heavy fermion superconductor CeRhln ${ }_{5}$. Nature 440, 65-68 (2006). 
5. Knebel, G., Aoki, D., Braithwaite, D., Salce, B. \& Flouquet, J. Coexistence of antiferromagnetism and superconductivity in $C$ Rhln $n_{5}$ under high pressure and magnetic field. Phys. Rev. B 74, 020501 (2006).

6. Löhneysen, Hv, Rosch, A. \& Vojta, M. Fermi-liquid instabilities at magnetic quantum phase transitions. Rev. Mod. Phys. 79, 1015-1075 (2007).

7. Monthoux, P., Pines, D. \& Lonzarich, G. G. Superconductivity without phonons. Nature 450, 1177-1183 (2007).

8. Gegenwart, P., Si, Q. \& Steglich, F. Quantum criticality in heavy-fermion metals. Nat. Phys. 4, 186-197 (2008).

9. Park, T. et al. Isotropic quantum scattering and unconventional superconductivity. Nature 456, 366-368 (2008).

10. Scalapino, D. J. A common thread: the pairing interaction for unconventional superconductors. Rev. Mod. Phys. 84, 1383-1417 (2012).

11. Nakai, Y. et al. Unconventional superconductivity and antiferromagnetism quantum critical behavior in the isovalent-doped $\mathrm{BaFe}_{2}\left(\mathrm{As}_{1-\mathrm{x}} \mathrm{P}_{\mathrm{x}}\right)_{2}$. Phys. Rev. Lett. 105, 107003 (2010).

12. Analytis, J. G. et al. Transport near a quantum critical point in $\mathrm{BaFe}_{2}\left(\mathrm{As}_{1-\mathrm{x}} \mathrm{P}_{\mathbf{x}}\right)_{2}$. Nat. Phys. 10, 194-197 (2014).

13. Keimer, B., Kivelson, S. A., Norman, M. R., Uchida, S. \& Zaanen, J. From quantum matter to high-temperature superconductivity in copper oxides. Nature 518, 179-186 (2015).

14. Jin, K., Butch, N. P., Kirshenbaum, K., Paglione, J. \& Greene, R. L. Link between spin fluctuations and electron pairing in copper oxide superconductors. Nature 476, 73-75 (2011).

15. Seo, S. et al. Controlling superconductivity by tunable quantum critical point. Nat. Commun. 6, 6433 (2015)

16. Keller, L. et al. Pressure dependence of the magnetic order in $\mathrm{CrAs}$ :A neutron diffraction investigation. Phys. Rev. B 91, 020409 (2014).

17. Shen, Y. et al. Structural and magnetic phase diagram of $\mathrm{CrAs}$ and its relationship with pressure-induced superconductivity. Phys. Rev. B 93, 060503 (2016).

18. Yu, Z. et al. Anomalous anisotropic compression behavior of superconducting CrAs under high pressure. Proc. Natl Acad. Sci. USA 112, 14766-14770 (2015).

19. $\mathrm{Wu}, \mathrm{W}$. et al. Superconductivity in the vicinity of antiferromagnetic order in CrAs. Nat. Commun. 5, 5508 (2014).

20. Kotegawa, H., Nakahara, S., Tou, H. \& Sugawara, H. Superconductivity of $2.2 \mathrm{~K}$ under pressure in helimagnet CrAs. J. Phys. Soc. Jpn. 83, 093702 (2014).

21. Kotegawa, $\mathrm{H}$. et al. Detection of an unconventional superconducting phase in the vicinity of the strong first-order magnetic transition in $\mathrm{CrAs}$ using ${ }^{75}$ As-nuclear quadrupole resonance. Phys. Rev. Lett. 114, 117002 (2015).
22. Matsuda, M. et al. Evolution of magnetic double helix and quantum criticality near a dome of superconductivity in CrAs. Phys. Rev. X 8, 031017 (2018).

23. Khasanov, R. et al. Pressure-induced electronic phase separation of magnetism and superconductivity in CrAs. Sci. Rep. 5, 13788 (2015).

24. Motizuki, K., Ido, H., Itoh, T. \& Mortifuji, M. Electronic structure and magnetism of 3d-transition metal pnictides. Springer Ser. Mater. Sci. 131, 1-142 (2009).

25. Rice, T. M., Barker, J. R. A. S., Halperin, B. I. \& McWHan, D. B. Antiferromagnetism in chromium and its alloys. J. Appl. Phys. 9, 1337-1343 (1969).

26. Jaramillo, R. et al. Breakdown of the Bardeen-Cooper-Schrieffer ground state at a quantum phase transition. Nature 459, 405-409 (2009).

27. Guo, $C$. Y. et al. Evidence for triplet superconductivity near an antiferromagnetic instability in CrAs. Phys. Rev. B 98, 024520 (2018).

28. Mackenzie, A. P. et al. Extremely strong dependence of superconductivity on disorder in $\mathrm{Sr}_{2} \mathrm{RuO}_{4}$. Phys. Rev. Lett. 80, 161-164 (1998).

29. Schnyder, A. P. \& Brydon, P. M. R. Topological surface states in nodal superconductors. J. Phys. Condens. Matter 27, 243201 (2015).

30. Wu, W. et al. Low temperature properties of pnictide CrAs single crystal. Sci. China Phys. Mech. Astron. 53, 1207-1211 (2010).

31. Eiling, A. \& Schilling, J. S. Pressure and temperature dependence of electrical resistivity of $\mathrm{Pb}$ and $\mathrm{Sn}$ from $1-300 \mathrm{~K}$ and $0-10 \mathrm{GPa}$-use as continuous resistive pressure monitor accurate over wide temperature range; superconductivity under pressure in $\mathrm{Pb}, \mathrm{Sn}$, and In. J. Phys. F Met. Phys. 11, 623-639 (1981).

(i) Open Access This article is licensed under a Creative Commons Attribution 4.0 International License, which permits use, sharing, adaptation, distribution and reproduction in any medium or format, as long as you give appropriate credit to the original author(s) and the source, provide a link to the Creative Commons license, and indicate if changes were made. The images or other third party material in this article are included in the article's Creative Commons license, unless indicated otherwise in a credit line to the material. If material is not included in the article's Creative Commons license and your intended use is not permitted by statutory regulation or exceeds the permitted use, you will need to obtain permission directly from the copyright holder. To view a copy of this license, visit http://creativecommons. org/licenses/by/4.0/.

(c) The Author(s) 2019 